\title{
Mixed Mullerian Tumor Primitive of Peritoneum: Report of a Case
}

\author{
Stefania Erra ${ }^{1}$, Simone Lo Mastro ${ }^{1}$, Ennio Nano ${ }^{1}$ \\ Surgical Pathology Department, Santo Spirito Hospital, Casale Monferrato (ITALY)
}

\section{ABSTRACT}

Carcinosarcoma is a rare and aggressive cancer that usually affects women in post menopausae; it represents $3 \%$ of all uterine neoplasms. Carcinosarcoma is a biphasic tumor characterized by a double component: a carcinomatous and a sarcomatous one, in variable amount of percentage from case to case. In the present article, a very rare case of primitive peritoneal carcinosarcoma is described in an 86-year-old woman, with senile atrophy of the uterus.

Keywords: Carcinosarcoma; mullerian mixed tumor; peritoneum.

\section{INTRODUCTION}

Carcinosarcoma or mullerian mixed tumor is a rare and aggressive type of tumor characterized by both mesenchymal and epithelial malignant cells. In most cases, uterus represents its primary site of origin, counting for about 3\% of all uterine neoplasms (Arend et al., 2011). The disease affects women in post menopausal age. The most affected area is the posterior wall of the uterus, in the fundus region (Barwick KW et al., 1979). As regards the carcinomatous component, it is typically glandular and if it is poorly differentiated the tumor is considered of high degree (Gersell DJ et al., 1989). On the basis of the kind of sarcomatous component, the neoplasia can be classified in to carcinosarcoma with heterologous component (reminiscent of other tissues typologies) or carcinosarcoma with homologous one when the sarcomatous component is due to stromal elements of the endometrial mucosa or uterine smooth muscle origin (Donner et al., 2003). Mullerian mixed tumor can give metastases and the degree of metastasis is more influenced by kind and percentage of the epithelial component (Emoto Met al., 1999). Among the genetic causes responsible for the tumor and which are investigable through immunohistochemistry are mutations of TP53, HER2 and S100 (Costa MJ et al., 1996).

\section{CASE REPORT}

In the present article a very rare case of a peritoneal carcinosarcoma is reported in an 86-year-old woman without uterine involvement. The patient has a negative pathological anamnesis, with no previous diseases. She never has been to the hospital not even to give birth. Some days before her first hospitalization, she began to feel stomach and abdominal pain. An abdominal ultrasound exam immediately showed a mass in the right hypochondrium, confirmed by a subsequent CT scan. The mass was surgically removed successfully with free margins of resection but the tumor has already metastasized and the woman is currently receiving palliative care for her advanced age and the late state of the disease. Surgical specimen has been analyzed in surgical pathology laboratory. Formalin fixed, paraffin embedded and Haematoxylin and Eosin stained slides have highlighted a biphasic tumor with low glandular endometrioid atypical pattern and abundant stromal atypical elements (Figure 1). Immunohistochemical analyzes have been performed confirming biphasic origin of the tumor, with Cytocheratin expression in glandular component (Figure 2) and underlining the presence of an homologous sarcomatous component, through immunohistochemical positivity for vimentin and S100 protein (Figure 3).

\section{DISCUSSION}

Carcinosarcoma accounts for only $2-3 \%$ of uterus tumors. It is a very aggressive malignancy for its poor differentiation and resistance towards standard cancer therapies, such as chemotherapy and radiotherapy, no effects have been seen against this pathology. Mullerian mixed tumor represents an high grade neoplasia.

\section{Corresponding author \\ Stefania Erra \\ Email : stefania.erra@virgilio.its}

Received: $21-11-2018$ 


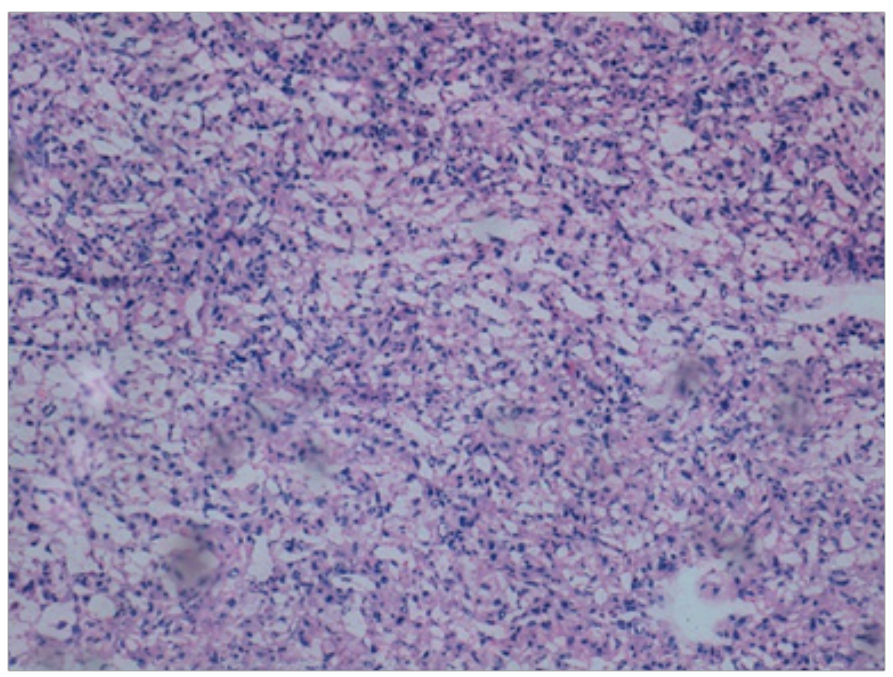

Figure. 1: EE- image of biphasic pattern of the tumor

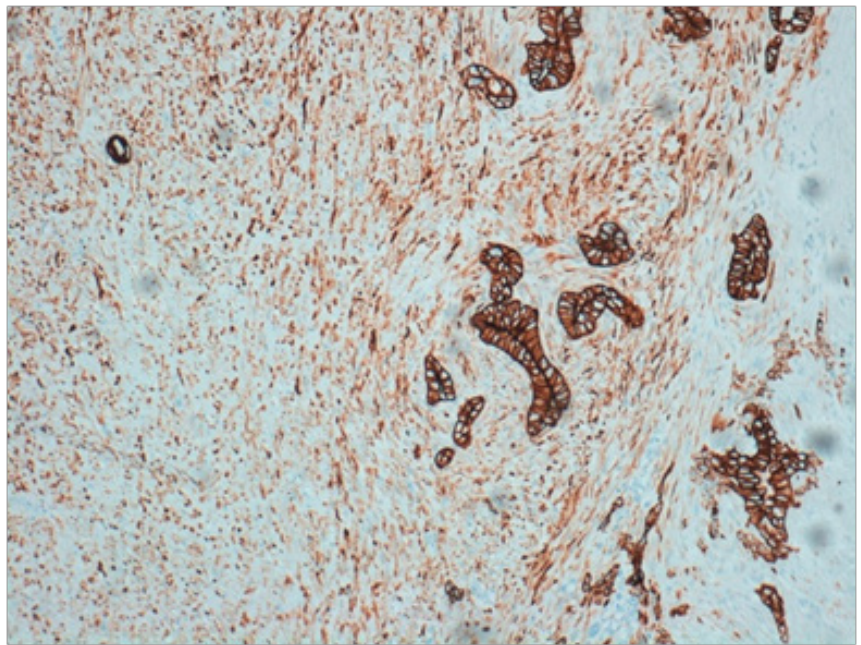

Figure 2: PanK positivity in glandular

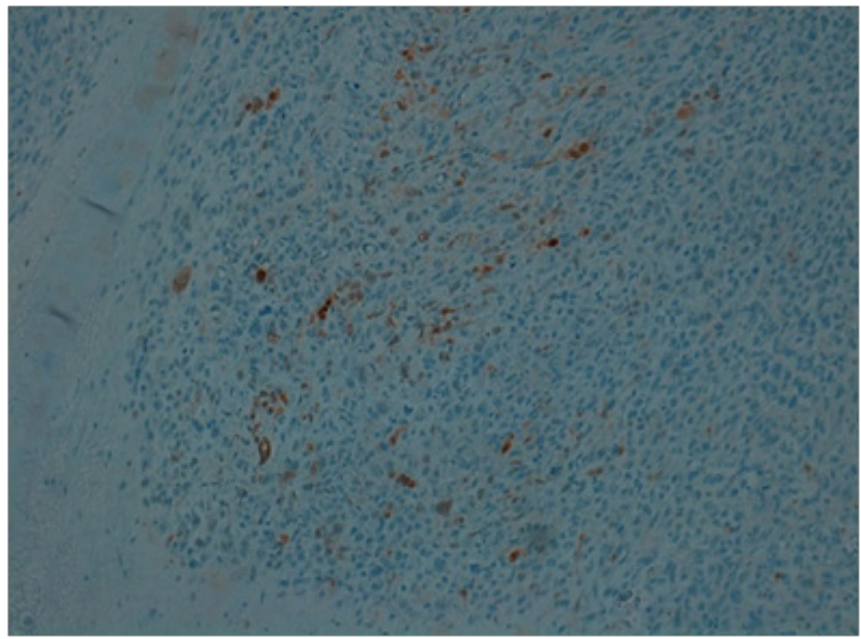

Figure. 3: S100 immunohistochemical positivity component
This means that it can spread to lymphnodes and other organs in high percentage of cases. Even in surgical well removed tumor, there is the probability of metastasis and relapse, maybe due to the microscopic proliferation of cancer cells. Despite carcinosarcoma's behavior, its diagnosis at an early stage (stage I) means an improved survival expectancy, which quickly drops after cancer proliferation. The symptoms of mixed mullerian tumor aren't specific. During cancer progression, there are compression and bleeding, sometimes with obstructions in the low abdomen and pelvic areas. Differential diagnosis of carcinosarcoma depends on the location of the primary tumor. Morphologically it can be distinguished from endometrial carcinoma when the mass originates from the uterine corpus, from mesothelioma and metastasis of other tumors if the origin site is the peritoneum. Surgical resection is the only curative option available for stage I patients, while it can be considered a palliative treatment for stage II and III. This tumor stadiation is easy on surgical specimen when tumor is in uterus, while for carcinosarcoma of the peritoneum to determine a correct staging is impossible (Ferguson SE et al., 2007). Etiologic factors predisponent for carcinosarcoma are not known, but there is some evidence that the tumor can be associated with the excessive use of estrogens and progesterone-containing contraceptives, like endometrial carcinoma (Wolfson AH et al., 2007). Histologically, mixed mullerian tumor is characterized by a double component, a stromal and an epithelial one. On the basis of the nature of the stromal component, it can be distinguished as an homologous carcinosarcoma, where it resembles mesenchymal tissue of the uterus or an undifferentiated mesenchymal tissue, and an heterologous carcinosarcoma, where the mesenchymal component resembles rhabdomyosarcomas and osteosarcomas. These last aspects are linked to a poorer prognosis (P Bitterman et al., 1990). Many studies shows that tumoral cells have many molecular changes, the most common being mutations of TP53, KRAS and PIK3CA (De Brito PA et al., 1993; Wada H et al., 1997). 


\section{CONCLUSION}

The case described can be considered very rare, because about thirty mixed mullerian tumor primitive of peritoneum have been reported in literature. Immunohistochemical profile of neoplastic elements is useful in differential diagnosis and in the definition of the type and percentage of the sarcomatous component. In fact, these data could have importance in biological behavior of the tumor, affecting overall survival and quality of life of the patients. As regards our case, the woman had a disease progression two months after the surgery, with evidence of multiple peritoneal nodular lesions in the CT examination.

\section{REFERENCES}

1. Arend Rebecca, Doneza Janine A, Wright Jason D (2011). Uterine Carcinosarcoma. Current Opinion in Oncology. 23(5):531-536.

2. Barwick KW, LiVolsi VA (1979). Malignant mixed mullerian tumors of the uterus. Amer. J. Surg. Pathol. 3:125-135.

3. De Brito PA, Silverberg SG, Orenstein JM (1993). Carcinosarcoma (malignant mixed mullerian mesodermal tumor) of the female genital tract. Immunoistochemical and ultrastructural analysis of 28 cases. Human pathology. 24:132-142.

4. Donner Ludvik R (2003). Uterine Carcinosarcoma with Complete Sarcomatous Overgrowth Mimicking Pure Embryonal Rhabdomyosarcoma. International Journal of Gynecological Pathology. 22(1):89-91.

5. Ferguson SE, Tornos C, Hummer A, Barakat RR, Soslow RA (2007). Prognostic features of surgical stage I uterine carcinosarcoma. Am J Surg Pathol. 31(11):1653-1661.
6. Gersell DJ, Duncan DA, Fulling KH (1989). Malignant mixed müllerian tumor of the uterus with neuroectodermal differentiation. Int. J. Gynecol. Pathol. 8:169-178

7. Makoto Emoto, Hiroshi Iwasaki, Masako Ishiguro, Masahiro Kikuchi, et al (1999). Angiogenesis in carcinosarcomas of the uterus: Differences in the microvessel density and expression of vascular endothelial growth factor between the epithelial and mesenchymal elements. Human Pathology. 30(10):1232-1241.

8. Michael J Costa, Judy Walls HT (1996). Epidermal growth factor receptor and c-erbB-2 oncoprotein expression in female genital tract carcinosarcomas (Malignant mixed müllerian tumors): Clinicopathologic study of 82 cases. Cancer: Publication cover image. 77(3):533-542.

9. P Bitterman, B Chun, Robert J Kurman (1990). The significance of epithelial differentiation in mixed mesodermal tumors of the uterus. A clinicopathologic and immunohistochemical study. American Journal of Surgical Pathology. 14(4):317-328.

10. Wolfson AH, Brady MF, Rocereto T, Mannel RS, Lee YC, et al (2007). A gynecologic oncology group randomized phase III trial of whole abdominal irradiation (WAI) vs. cisplatinifosfamide and mesna (CIM) as post-surgical therapy in stage I-IV carcinosarcoma (CS) of the uterus. GynecolOncol. 107(2):177-185.

11. Wada H, Enomoto T, Fujita M, Yoshino K, et al (1997). Molecular evidence that most but not all carcinosarcomas of the uterus are combination tumors. Cancer Res. 57(23):5379-5385.

How to cite article: Stefania Erra, Simone Lo Mastro, Ennio Nano (2018). A rare case fo male breast metastasis from previously removed renal cell carcinoma. Int. J. Appl. Pharm. Sci. Res. 4(1):1-3. doi: https://doi.org/10.21477/ ijapsr.4.1.1

Source of Support; Nil Conflict of Interest: None declared 